PROCEEDINGS OF THE

AMERICAN MATHEMATICAL SOCIETY

Volume 133, Number 12, Pages 3647-3655

S 0002-9939(05)07981-5

Article electronically published on June 2, 2005

\title{
BAND LIMITED FUNCTIONS ON QUANTUM GRAPHS
}

\author{
ISAAC PESENSON
}

(Communicated by David R. Larson)

\begin{abstract}
The notion of band limited functions is introduced on a quantum graph. The main results of the paper are a uniqueness theorem and a reconstruction algorithm of such functions from discrete sets of values. It turns out that some of our band limited functions can have compact supports and their frequencies can be localized on the "time" side. It opens an opportunity to consider signals of a variable band width and to develop a sampling theory with variable rate of sampling.
\end{abstract}

\section{INTRODUCTION}

The classical sampling theorem says that if the Fourier transform of a function $f \in L_{2}\left(\mathbb{R}^{1}\right)$ has support in $[-\omega, \omega]$, then $f$ is completely determined by its values at points $n \Omega$, where $\Omega=\pi / \omega$ and in $L_{2}$-sense

$$
f(t)=\sum f(n \Omega) \frac{\sin (\omega(t-n \Omega))}{\omega(t-n \Omega)} .
$$

Band limited functions are also uniquely determined and can be recovered from their values on some irregular sets of points $x_{j}$. As was shown by Paley and Wiener 12 it is enough to assume that functions $\exp i x_{j} t, j \in Z$, form a Riesz basis for $L_{2}([-\pi, \pi])$.

In the present article the notion of band limited functions is introduced on a general quantum graph. The main results of the paper are a uniqueness theorem (Theorem 4.1) that says that band limited functions on quantum graphs are completely determined by their values on some discrete sets of points and a reconstruction algorithm (Theorem 4.3) which gives a way for reconstruction of such functions from discrete sets of values.

Quantum graphs found numerous applications in physics, chemistry, engineering and quantum computing. They serve as models in many situations when one deals with waves that propagate in "thin" media. Basic notions and further references on this subject can be found in [3], [6, 7], 10].

It is known that the classical approach to the signal analysis by using the language of band limited functions [17] has serious drawbacks. By the Paley-Wiener theorem every band limited function is an entire function of exponential type, and for this reason cannot have "too many" zeros. In particular it means that a nonzero band limited signal cannot terminate in time. As a consequence of this there

Received by the editors May 4, 2004 and, in revised form, August 20, 2004.

2000 Mathematics Subject Classification. Primary 94A12, 05C99; Secondary 47E05.

Key words and phrases. Quantum graphs, band limited functions, reconstruction algorithm. 
is another problem: it is impossible to "separate" frequencies by saying that a band limited function has one band width during one interval of time and a different band width during another time interval. Both of these problems can also be viewed as consequences of the uncertainty principle.

It seems very interesting that in the case of quantum graphs there are non-zero band limited functions with compact supports. By this we mean that there are nonzero band limited functions on a graph which are identical to zero on some of its subgraphs. As a result, linear combinations of such functions will represent band limited functions of "variable" band width in the sense that they will have one band width as functions on one subgraph and a different band width as functions on other subgraphs.

The basic notion of band limitedness is introduced in the second section. We consider a quantum graph $(\Gamma, \Delta)$, where $\Gamma$ is a metric graph and $\Delta$ is a Hamiltonian on $\Gamma$, which acts on each edge as the (negative) second derivative, and the description of the domain of $\Delta$ involves the Kirchhoff (Neumann) compatibility conditions. It is a self-adjoint positive definite operator in the corresponding space $L_{2}(\Gamma)$, and we say that a function is band limited with band width $\omega$ if its image in the spectral representation of $\Delta$ has compact support in the interval $[0, \omega]$. We give some equivalent descriptions of such band limited functions which show that they enjoy some of the main properties of the classical band limited function. For example, they are analytic vectors for the self-adjoint operator $\Delta$ and can be identified with entire vector-valued functions which have exponential type and which are bounded on the real line.

In the third section we construct different examples of band limited functions with compact supports.

In the last section, section 4 , we prove our uniqueness result and the reconstruction algorithm. Namely, the uniqueness theorem, Theorem 4.1, says that every $\omega$-band limited function on $\Gamma$ is completely determined by its values on appropriate countable sets of points if the distance between points is not bigger than $\pi / \omega$. Theorem 4.3 shows how to reconstruct $\omega$-band limited functions from their values on such sets by using an iteration procedure which is also known as the Voronoi method.

Note that the same method was used by R. Coifman and R. Rochberg in 2 for reconstruction of analytic functions. Their paper inspired H. Feichtinger and C. Grochening [4, who used this method for reconstruction of band limited functions from the space $L_{2}\left(\mathbb{R}^{1}\right)$. The latter result was generalized by H. Feichtinger and I. Pesenson [5] to the case of a Riemannian manifold of bounded geometry.

\section{QUANTUM GRAPHS AND BAND LIMITED FUNCTIONS}

A quantum graph is a pair consisting of a metric graph $\Gamma$ and a self-adjoint differential operator on it.

Recall that a metric graph is a set of vertices $V=\left\{v_{i}\right\}$ and edges $E=\left\{e_{i}\right\}$ each of length $\left|e_{i}\right| \in(0, \infty]$. We identify every edge $e$ with a segment $[0,|e|]$ of $R^{1}$ and use coordinate $x_{e}$ along it. If the graph $\Gamma$ is infinite, the common assumption is that the length of all edges is bounded from below

$$
|e| \geq a>0, e \in E .
$$

The graph $\Gamma$ can be equipped with a natural metric and the Lebesgue measure $d x$. 
We will use the notation $E_{v}$ for the set of all edges containing $v$ as a vertex, and we assume that all these sets are finite. We allow the existence of infinite edges and do not restrict a number of such edges; however, we assume that the length of all finite edges is bounded from above

$$
e \in E_{c} \Rightarrow|e| \leq b,
$$

where $E_{c}$ is a set of all finite edges and a constant $b$ is not less than the constant $a$ from (2.1).

The space $L_{2}(\Gamma)$ is defined as the direct sum of spaces $L_{2}(e), e \in E$, with the scalar product

$$
\langle f, g\rangle=\sum_{e \in E} \int_{e} f \bar{g} d x, f, g \in L_{2}(\Gamma)
$$

and the norm

$$
\|f\|_{L_{2}(\Gamma)}=\left(\sum_{e \in E} \int_{e}|f|^{2} d x\right)^{1 / 2} .
$$

There are many ways to introduce a self-adjoint operator on $\Gamma$ which is called a Hamiltonian. In this paper we will deal with the operator which acts along each edge as the negative second derivative

$$
-\frac{d^{2}}{d x^{2}}
$$

and at every vertex $v$ satisfies the so-called Neumann (Kirchhoff) conditions

$$
\sum_{e \in E_{v}} \frac{d f}{d x}(v)=0
$$

where $E_{v}$ is the set of all edges containing $v$ as a vertex and the derivatives are taken in the directions away from the vertex.

Definition 1. The Hamiltonian $\Delta$ is defined by formula (2.2) on each edge $e \in E$ and its domain $\mathcal{D}(\Delta)$ consists of all continuous functions from $L_{2}(\Gamma)$ that satisfy boundary conditions (2.3) and belong to the Sobolev space $H^{2}(e)$ on each edge $e$. The domain $\mathcal{D}(\Delta)$ is treated as the Sobolev space $H^{2}(\Gamma)$ equipped with the graph norm

$$
\|f\|_{H^{2}(\Gamma)}=\left(\sum_{e \in E} \int_{e}\left(|f|^{2}+\left|\frac{d^{2} f}{d x^{2}}\right|^{2}\right) d x\right)^{1 / 2} .
$$

It is known that such a defined operator $\Delta$ is self-adjoint and positive definite in the space $L_{2}(\Gamma)$. In particular its spectrum belongs to the interval $[0, \infty]$. If $P_{t}$ is the associated spectral resolution of the identity, then

$$
\Delta f=\int_{0}^{\infty} t d P_{t} f, f \in L_{2}(\Gamma) .
$$

Using the spectral resolution of identity we define the unitary group of operators by the formula

$$
e^{i t \Delta} f=\int_{0}^{\infty} e^{i t} d P_{t} f, f \in L_{2}(\Gamma), t \in \mathbb{R}
$$


Definition 2. We say that a function $f \in L_{2}(\Gamma)$ is band limited with band width $\omega$ or $\omega$-band limited if the corresponding measure $d P_{t} f$ has support in $[0, \omega]$. The set of all $\omega$ band limited functions will be denoted by $E^{\omega}=E^{\omega}(\Gamma)$.

An equivalent definition of the spaces $E^{\omega}(\Gamma)$ can be given in terms of the following representation of a self-adjoint operator $\Delta$ which is some kind of the abstract Fourier transform. Note that such a "Fourier" representation of a self-adjoint operator is not unique.

According to the spectral theory [1 for a self-adjoint positive definite operator $\Delta$ in a Hilbert space $L_{2}(\Gamma)$, there exist a direct integral of Hilbert spaces $X=$ $\int X(\lambda) d m(\lambda)$ and a unitary operator $F$ from $L_{2}(\Gamma)$ onto $X$, which transforms the domain of $\Delta^{\mu}, \mu \geq 0$, onto $X_{\mu}=\left\{x \in X \mid \lambda^{\mu} x \in X\right\}, X_{0}=X$, with the norm

$$
\|x(\lambda)\|_{X_{\mu}}=\left(\int_{0}^{\infty} \lambda^{2 \mu}\|x(\lambda)\|_{X(\lambda)}^{2} d m(\lambda)\right)^{1 / 2} .
$$

Besides,

$$
F\left(\Delta^{\mu} f\right)=\lambda^{\mu}(F f)
$$

if $f$ belongs to the domain of $\Delta^{\mu}$.

Definition 3. We will say that a function $f$ from $L_{2}(\Gamma)$ is $\omega$-band-limited if its "Fourier transform" $F f$ has support in $[0, \omega]$. Then $E^{\omega}(\Gamma)$ will denote the set of all $\omega$-band-limited vectors.

The following results can be found in [13].

Theorem 2.1. Let the $\mathcal{D}\left(\Delta^{k}\right), k \in \mathbb{N}$, be the domain of the operator $\Delta^{k}$ and $\mathcal{D}^{\infty}=$ $\bigcap_{k \in \mathbb{N}} \mathcal{D}\left(\Delta^{k}\right)$. The following hold true:

(1) the set $\bigcup_{\omega>0} E^{\omega}(\Gamma) \subset \mathcal{D}^{\infty}$ is dense in $L_{2}(\Gamma)$;

(2) the set $E^{\omega}(\Gamma)$ is a linear closed subspace in $L_{2}(\Gamma)$;

(3) $f \in E^{\omega}(\Gamma)$ if and only if for all $s \geq 0$ the following Bernstein inequality holds true:

$$
\left\|\Delta^{s} f\right\|_{L_{2}(\Gamma)} \leq \omega^{s}\|f\|_{L_{2}(\Gamma)} ;
$$

(4) $f \in E^{\omega}(\Gamma)$ if and only if for every $g \in L_{2}(\Gamma)$ the scalar-valued function of the real variable $t \in \mathbb{R}^{1}$

$$
\left\langle e^{i t \Delta} f, g\right\rangle=\sum_{e \in E} \int_{e} e^{i t \Delta} f \bar{g} d x
$$

is bounded on the real line and has an extension to the complex plain as an entire function of the exponential type $\omega$.

Note that to prove (1), (2) and (3) one has to use the formulas (2.4) and (2.6). Property (4), which is a variant of the Paley-Wiener theorem, is essentially a consequence of (3) which implies that for a function $f$ from $E^{\omega}(\Gamma)$ the exponential series

$$
e^{i z \Delta} f=\sum_{k=0}^{\infty} \frac{(i z \Delta)^{k} f}{k !}, z \in \mathbb{C},
$$

is absolutely convergent everywhere and

$$
\left\|e^{i z \Delta} f\right\|_{L_{2}(\Gamma)} \leq e^{\omega|z|}\|f\|_{L_{2}(\Gamma)}, z \in \mathbb{C} .
$$


In the case of a finite graph $\Gamma$ the spectrum of the Hamiltonian operator is discrete, non-negative and goes to infinity. In this case the space $E^{\omega}(\Gamma)$ consists of all linear combinations of eigenfunctions whose eigenvalues are not greater than $\omega$.

It is clear that for any graph $\Gamma$ functions from $E^{\omega}(\Gamma)$ are infinitely differentiable inside of any edge. Moreover they are real analytic functions on any open edge. Indeed, every $f \in E^{\omega}(\Gamma)$ is an analytic vector of the differential operator $\Delta$ on any open edge (which means that the series (2.7) is convergent). By the theorem of Nelson [11] it implies that the function $f$ is real analytic on any open edge.

\section{EXAMPLES OF BAND LIMITED FUNCTIONS WITH COMPACT SUPPORTS ON GRAPHS}

The goal of this section is to demonstrate that the spaces of band limited functions $E^{\omega}(\Gamma)$ contain functions with compact supports. As a consequence some band limited functions can be represented as linear combinations of functions which can be "localized" in frequency space as well as in "time" space.

Example 1. Suppose that there is a path $v_{0}, v_{1}, \ldots, v_{k}=v_{0}$ (a loop) from a vertex $v_{0}$ to $v_{0}$. We identify this path with a circle and consider an eigenfunction $\psi$ of the second derivative on this circle which has zeros at the points $v_{0}, v_{1}, \ldots, v_{l}=v_{0}$. Then the new function $\varphi$ (which is an extension of this $\psi$ by zero to the rest of the graph) does belong to the domain of any natural power of the Hamiltonian $\Delta$. Moreover, if the eigenfunction $\psi$ corresponds to the eigenvalue $\omega$, then

$$
\left\|\Delta^{k} \varphi\right\|_{L_{2}(\Gamma)} \leq \omega^{k}\|\varphi\|_{L_{2}(\Gamma)} .
$$

Example 2. Suppose that a graph $\Gamma$ contains several loops $l_{1}, l_{2}, \ldots, l_{N}$. Let $\psi_{j}, j=$ $1,2, \ldots, N$, be an eigenfunction of the second derivative

$$
-\frac{d^{2} \psi_{j}}{d x^{2}}=\omega_{j} \psi_{j}
$$

on the loop $l_{j}, j=1,2, \ldots, N$, which has zeros at all vertices of the loop $l_{j}, j=$ $1,2, \ldots, N$. Then a function $\varphi$ which coincides with $\psi_{j}$ on each $l_{j}$ and is zero everywhere else belongs to the space $E^{\omega}(\Gamma)$, where $\omega=\max \left(\omega_{1}, \omega_{2}, \ldots, \omega_{N}\right)$.

Example 3. As another example let us consider $X$-axis and $Y$-axis as infinite edges of a metric graph $\Gamma$ whose only "finite" vertex is the origin. Take a function $\psi_{1}$, which is the inverse Fourier transform of an odd function $\widehat{\psi_{1}}$ on an interval $\left[-\omega_{1}, \omega_{1}\right]$, and a function $\psi_{2}$, which is the inverse Fourier transform of an odd function $\widehat{\psi_{2}}$ on an interval $\left[-\omega_{2}, \omega_{2}\right]$.

Introduce a function $\varphi_{1}$ which is $\psi_{1}$ on the $X$-axis and zero on the $Y$-axis. It is a function which belongs to the domain of any power $k \in \mathbb{N}$ of $\Delta$ and

$$
\left\|\Delta^{k} \varphi_{1}\right\|_{L_{2}(\Gamma)} \leq \omega_{1}^{2 k}\left\|\varphi_{1}\right\|_{L_{2}(\Gamma)} .
$$

Thus, $\varphi_{1} \in E^{\omega_{1}}(\Gamma)$ and is zero on the $Y$-axis.

In a similar way we can introduce the function $\varphi_{2} \in E^{\omega_{2}}(\Gamma)$ which has support on the $Y$-axis, i.e. it is $\psi_{2}$ on the $Y$-axis and zero on the $X$-axis.

It is easy to verify that any linear combination of $\varphi_{1}$ and $\varphi_{2}$ belongs to the domain of every power $\Delta^{k}, k \in \mathbb{Z}$, and since they are orthogonal their linear combination $\vartheta$ 
will satisfy

$$
\left\|\Delta^{k} \vartheta\right\|_{L_{2}(\Gamma)} \leq \omega^{2 k}\|\vartheta\|_{L_{2}(\Gamma)}
$$

where $\omega=\max \left(\omega_{1}, \omega_{2}\right)$.

Note that all these examples can be generalized in many different ways.

\section{A Uniqueness THEOREM AND A RECONSTRUCtion ALGORITHM}

The goal of this section is to prove that band limited functions are uniquely determined and can be reconstructed in a stable way from their values on sufficiently dense sets of points of a quantum graph.

In the rest of the paper we will use the notation $I(\rho)=\left\{I_{j}\right\}$ for the so-called admissible covers of $\Gamma$ that satisfy the following definition.

Definition 4. We say that a set $I(\rho)=\left\{I_{j}\right\}$ of open and pairwise disjoint intervals $I_{j}$ is an admissible cover of $\Gamma$ if it has the following properties:

1) the union of all open intervals $I_{j}$ does not contain vertices of $\Gamma$;

2 ) the union of closures of all intervals $I_{j}$ is a cover of $\Gamma$;

3) there exists a positive $\rho$ such that

$$
\left|I_{j}\right| \leq \rho \leq \inf _{e \in E_{c}}|e|,
$$

where $E_{c}$ is the set of all edges of finite length.

Note that the set of all end points of all intervals $I_{j}$ contain all vertices of the graph $\Gamma$.

Theorem 4.1. Any function $f$ from a space $E^{\omega}(\Gamma), \omega>0$, is uniquely determined by its values at the end points of the intervals $I_{j}$ which form an admissible cover $I(\rho)=\left\{I_{j}\right\}$ of the graph $\Gamma$ with

$$
0<\rho<\frac{\pi}{\sqrt{\omega}}
$$

Proof. We will need the following generalization of Wirtingen's inequality (see 9], [16] for proofs). If a function $f$ is zero at the end points of an interval $I_{j}$ and $f \in H^{2}\left(I_{j}\right)$, then

$$
\|f\|_{L_{2}\left(I_{j}\right)} \leq\left(\frac{\left|I_{j}\right|}{\pi}\right)^{2}\left\|f^{\prime \prime}\right\|_{L_{2}\left(I_{j}\right)} \leq\left(\frac{\rho}{\pi}\right)^{2}\left\|f^{\prime \prime}\right\|_{L_{2}\left(I_{j}\right)} .
$$

It shows that if $f \in H^{2}(\Gamma)$, then

$$
\|f\|_{L_{2}(\Gamma)} \leq\left(\frac{\rho}{\pi}\right)^{2}\|\Delta f\|_{L_{2}(\Gamma)} .
$$

This inequality along with the Bernstein inequality (2.6) for functions from $E^{\omega}(\Gamma)$ give

$$
\|f\|_{L_{2}(\Gamma)} \leq\left(\frac{\rho}{\pi}\right)^{2} \omega\|f\|_{L_{2}(\Gamma)} .
$$

The last inequality and the assumption (4.1) imply the uniqueness result.

It turns out that the band limited functions from $E^{\omega}(\Gamma)$ can be reconstructed from their values at end points of the intervals $I_{j}$. 
Lemma 4.2. Let $S$ be a bounded operator in a Hilbert space $H$ such that for some $\alpha<1$ and for all $f \in H$

$$
\|f-S f\| \leq \alpha\|f\| .
$$

Then $S$ is invertible and $f$ can be recovered from $S f$ by the following iterative procedure. If $f_{0}=S f$ and

$$
f_{n+1}=f_{n}+S\left(f-f_{n}\right)
$$

then

$$
\lim _{n \rightarrow \infty} f_{n}=f
$$

with the error estimate

$$
\left\|f-f_{n}\right\| \leq \alpha^{n+1}\|f\|
$$

Proof. Since the norm of the operator $I-S$ is less than 1, operator $S$ is invertible and the inverse $S^{-1}$ can be represented by a Neumann series

$$
S^{-1}=\sum_{n=0}(I-S)^{n} .
$$

Thus, every $f \in H$ is determined by $S f$ because

$$
f=S^{-1} S f=\sum_{n=0}^{\infty}(I-S)^{k} S f=\lim _{n \rightarrow \infty}\left(\sum_{n=0}^{n}(I-S)^{n} S f\right) .
$$

The sequence $f_{n}$ of partial sums

$$
f_{n}=\sum_{k=0}^{n}(I-S)^{k} S f
$$

satisfies the stated recursion relation (4.5). The identity

$$
\sum_{k=n+1}^{\infty}(I-S)^{k}=(I-S)^{n+1} S^{-1}
$$

and the assumption (4.4) imply

$$
\left\|f-f_{n}\right\|=\left\|\sum_{k=n+1}^{\infty}(I-S)^{k} S f\right\|=\left\|(I-S)^{n+1} S^{-1} S f\right\| \leq \alpha^{n+1}\|f\| .
$$

Suppose that a function $f \in E^{\omega}(\Gamma), \omega \geq 0$, is known at the end points $\left(a_{j}, b_{j}\right)$ of intervals $I_{j}$ which constitute an admissible cover $I(\rho)$ of the graph $\Gamma$. Clearly, $b_{j}=a_{j+1}$. Thus we assume that there exist a function $f \in E^{\omega}(\Gamma), \omega \geq 0$, and a set of its values $\left\{f\left(a_{j}\right)\right\} \in l_{2}$ at the points $\left\{a_{j}\right\}$ is given. Let $F_{f}$ be a linear function which is defined by the formula

$$
F_{f}(x)=f\left(a_{j}\right)+\frac{f\left(b_{j}\right)-f\left(a_{j}\right)}{b_{j}-a_{j}}\left(x-a_{j}\right), x \in I_{j}=\left(a_{j}, b_{j}\right) .
$$

Let

$$
P_{\omega}: L_{2}(\Gamma) \rightarrow E^{\omega}(\Gamma)
$$

be the orthogonal projector. Using this notation we can formulate our reconstruction algorithm. 
Theorem 4.3. If

$$
0<\rho<\frac{\pi}{\sqrt{\omega}}
$$

then for any admissible cover $I(\rho)$ every function $f$ from $E^{\omega}(\Gamma)$ can be obtained from a sequence $\left\{f\left(a_{j}\right)\right\} \in l_{2}$ as the following limit in the $L_{2}(\Gamma)$-norm:

$$
f=\lim _{n \rightarrow \infty} f_{n}
$$

of the functions $f_{n}$, where

$$
f_{0}=P_{\omega} F_{f}
$$

and

$$
f_{n+1}=f_{n}+P_{\omega} F_{\left(f-f_{n}\right)}, n \in \mathbb{N} .
$$

The error estimate in the convergence (4.11) is

$$
\left\|f-f_{n}\right\|_{L_{2}(\Gamma)} \leq\left(\frac{\rho \sqrt{\omega}}{\pi}\right)^{2(n+1)}\|f\|_{L_{2}(\Gamma)} .
$$

Proof. Since $P_{\omega}$ is the orthogonal projector on $E^{\omega}(\Gamma)$ and $f \in E^{\omega}(\Gamma)$ we have for $S f=P_{\omega} F_{f}$

$$
\begin{aligned}
& \|f-S f\|_{L_{2}(\Gamma)}^{2}=\left\|P_{\omega}\left(f-F_{f}\right)\right\|_{L_{2}(\Gamma)}^{2} \leq\left\|f-F_{f}\right\|_{L_{2}(\Gamma)}^{2} \\
& \leq \sum_{j} \int_{a_{j}}^{b_{j}}\left|f(x)-f\left(a_{j}\right)-\frac{f\left(b_{j}\right)-f\left(a_{j}\right)}{b_{j}-a_{j}}\left(x-a_{j}\right)\right|^{2} d x .
\end{aligned}
$$

An application of the inequality (4.3), assumption (4.10) and the Bernstein inequality (2.6) imply the theorem.

\section{REFERENCES}

1. M. Birman and M. Solomyak, Spectral thory of selfadjoint operators in Hilbert space, D. Reidel Publishing Co., Dordrecht, 1987. MR.1192782 (93g:47001)

2. R. Coifman and R. Rochberg, Representation theorems for holomorphic and harmonic functions in $L^{p}$, Asterisque 77, (1980), 11-66. MR0604369 (82j:32015)

3. W.D. Evans and D.J. Harris, Fractals, trees, and the Neumann Laplacian, Math. Ann. 296, (1993), 493-527. MR 1225988 (94k:35218)

4. H. Feichtinger and K. Gröchenig, Theory and practice of irregular sampling in Wavelets: mathematics and applications (J.J. Benedetto, M.W. Frazier, editors), Stud. Adv. Math., CRC, Boca Raton, FL, 1994, 305-363. MR 1247520 (94i:94008)

5. H. Feichtinger and I. Pesenson, Iterative recovery of band limited functions on manifolds, in Wavelets, Frames and Operator Theory, (C. Heil, P.E.T. Jorgensen, D.R. Larson, editors), Contemp. Math., 345, AMS (2004), 137-153. MR2066825

6. V. Kostrykin and R. Schrader, Kirchoff's rule for quantum wires, J. Phys. A 32, (1999), 595-630. MR1671833 (99m:81280)

7. V. Kostrykin and R. Schrader, Kirchoff's rule for quantum wires:II The inverse problem with possible applications to quantum computers, Fortschrt. Phys. 48, (2000), 703-716. MR 1778728 (2001g:81273)

8. P. Kuchment, Quantum graphs: Some basic structures, Waves Random Media 14 (2004), 107-128. MR2042548

9. Ky Fan, O. Taussky, and J. Todd, Disrete analogs of inequalities of Wirtingen, Monatsh. Math. 59 (1955), 73-90. MR0070676 (17:19b)

10. K. Naimark and M. Solomyak, Egenvalue estimates for the weighted Laplacian on metric trees, Proc. London Math. Soc.(3) 80 (2000), 690-724. MR1744781 (2002c:47085)

11. E. Nelson, Analytic vectors, Ann. of Math. 70(3), (1959), 572-615. MR0107176 (21:5901)

12. R.E.A.C. Paley and N. Wiener, Fourier Transforms in the Complex Domain, Coll. Publ. 19, Providence: Amer. Math. Soc., (1934), reprint, 1987. MR1451142 (98a:01023) 
13. I. Pesenson, A sampling theorem on homogeneous manifolds, Trans. of AMS 352(9), (2000), 4257-4270. MR 1707201 (2000m:41012)

14. I. Pesenson, Sampling of Band limited vectors, J. of Fourier Analysis and Applications 7(1), (2001), 93-100. MR1812998 (2002b:42034)

15. I. Pesenson, Poincare-type inequalities and reconstruction of Paley-Wiener functions on manifolds, J. of Geometric Analysis 14(1), (2004), 101-121. MR2030577 (2004h:42030)

16. I. Pesenson, Reconstruction of band-limited functions in $L_{2}\left(R^{d}\right)$, Proceed. of AMS, 127(12), (1999), 3593- 3600. MR1610773 (2000f:42002)

17. C. Shannon and W. Weaver, The Mathematical Theory of Communication, Univ.of Illinois Press, 1963. MR0032134(11:258e)

Department of Mathematics, Temple University, Philadelphia, Pennsylvania 19122

E-mail address: pesenson@math.temple.edu 